\title{
Recidiva bioquímica precoz tras prostatectomía radical retropúbica por cáncer de próstata. Factores implicados. ¿Influye el tiempo de espera entre biopsia y cirugía?
}

\author{
Blanco Díez A, Armas Molina J, Limiñana Canal JMª, Jiménez García C, Artíles Hernández JL, \\ Chesa Ponce N.
}

Servicio de Urologia. *Unidad de Investigación. Hospital Universitario Insular de Gran Canaria. Las Palmas de Gran Canaria.

Actas Urol Esp. 2008;32(4):406-410

\begin{abstract}
RESUMEN
RECIDIVA BIOQUÍMICA PRECOZ TRAS PROSTATECTOMÍA RADICAL RETROPÚBICA POR CÁNCER DE PRÓSTATA. FACTORES IMPLICADOS. ¿INFLUYE EL TIEMPO DE ESPERA ENTRE BIOPSIA Y CIRUGÍA?

Objetivos: Estudiar diferentes factores que pueden influir en la recidiva bioquímica precoz (RBP) (primer año tras cirugía), tras prostatectomía radical retropúbica (PRR).

Material y métodos: Estudiamos 310 PRR. Criterios de inclusión: al menos un año de seguimiento, ausencia de bloqueo hormonal o radioterapia previa. Los pacientes fueron divididos inicialmente en 2 grupos según el tiempo de espera. Grupo A <90 días (n: 148), Grupo B > 90 días (n:162). Estudiamos RBP ( 2 o más determinaciones de PSA >= 0,2 ng/ml) y la influencia del PSA previo, el Gleason de la biopsia, el Gleason del espécimen, estadio pT, y tiempo de espera. Para estudiar la homogeneidad existente entre los dos grupos usamos la t de Student o W de Wilcoxon. Estudiamos la RBP y el estadio pT en los dos grupos usando la chi cuadrado de Pearson, que también nos sirve para estudiar en los dos grupos la RBP en relación al estadio pT. El test de la U de mann-Whitney lo usamos para estudiar en la serie global la RBP según el Gleason del espécimen. Por último se realiza una Regresión logística multivariante para estudiar la influencia de todas las variables en la RBP en la serie global.

Resultados: No encontramos diferencias entre los dos grupos en edad ( $p<0,129)$, ni PSA ( $<<0,479)$, ni Gleason de biopsia $(p<0,913)$. No se encontraron diferencias en RBP ni en estadio pT según el tiempo de espera. Hallamos diferencias estadísticamente significativas en RBP si estudiamos estadio pT y Gleason de la pieza. Los T3 tienen más incidencia de recurrencia que los T2 y hay más incidencia de RBP según aumenta el Gleason de la pieza. En la regresión logística de la serie global las variables independientes de progresión son: PSA previo y estadio pT. Ni el Gleason de la biopsia ni el Gleason del espécimen, ni el tiempo de espera entre biopsia y cirugía influyen en la RBP.

Conclusiones: El Gleason de la biopsia y el tiempo de espera no influyen en la RBP. El tiempo de espera tampoco influye en el estadio T final. En nuestra serie las diferencias en RBP vienen dadas por el PSA previo y pT. El Gleason del espécimen parece influir en la RBP, pero en menor proporción sin significado en el análisis multivariante.

Palabras clave: Cáncer de próstata. Recurrencia. Biopsia de próstata.
\end{abstract}

\section{ABSTRACT}

EARLY BIOCHEMICAL FAILURE AFTER RADICAL PROSTATECTOMY FOR PROSTATE CANCER. IMPACT FACTORS?

Does delay from biopsy to surgery have any influence?Objectives: To determine wether WT may impact on the EBF (before one year) alter RRP in our series, and to study other factors that may impact on this issue.

Material and methods: We study 310 RRP. Inclusion criteria are: one year follow up, no hormonal manipulation neither previous radiotherapy. Patients are divide in two groups attending WT. Group A < 90 days (n: 148), Group B > 90 days (n: 162). We study EBF ( 2 or more PSA determinations $>=0.2 \mathrm{ng} / \mathrm{ml}$ ), and the impact of previous PSA, biopsy Gleason, Gleason of the specimen (G), pT stage (pT), and WT. T of Student or W of Wilcoxon are used to determine the homogeneity of the two groups. Chi Square of Pearson to compare the two group's EBF and pT attending to WT and EBF attending to pT. U of Mann Whitney is used to study EBF attending to G. Multivariate logistic regresion (LR) is used to study the impact of the variables on EBF.

Results: There are no differences between the two groups in age ( $p<0.129)$, PSA ( $p<0.479)$, biopsy“s Gleason $(p<0.913)$. There are no differences in EBF nor in pT attending to WT. Significant statystical differences are founded in EBF attending to pT and G. T3 tumors have more chance of recurrence than T2. EBF is more frecuent as G increases.

In logistic regresion of the global serie, independent variables for progression are: Previous PSA and pT. Nor biopsy`s Gleason nor specimen's Gleason, nor WT, impact on the EBF

Conclusions: Biopsy Gleason and WT do not impact on EBF. WT do not impact on pT. In our serie differences on EBF are lead by previous PSA and pT. Also Gleason of the especimen seems to impact on EBF, but in lower proportion, with no significance in multivariate analysis.

Keywords: Prostate cancer. Recurrence. Prostate biopsy. 
$\mathrm{D}$ esde finales de los años ochenta ha habido cambios importantes en lo que respecta al abordaje del cáncer de próstata. La disponibilidad del PSA nos ha permitido diagnosticar esta enfermedad en estadios iniciales. A esto se ha asociado un mayor conocimiento anatómico que nos ha permitido perfeccionar el abordaje quirúrgico obteniendo mejores tasas de curación con menos efectos indeseables derivados de dicho abordaje. La prostatectomía radical se ha convertido en una de las principales opciones para el tratamiento del cáncer de próstata localizado, y suele realizarse en pocos meses tras el diagnóstico con biopsia. En algunos países europeos como España el Sistema Público de Salud nos fuerza a tener listas de espera antes de la cirugía. Esto puede presentar un problema para el paciente por la tensión que supone el saberse en espera por una patología maligna. En este trabajo estudiamos nuestra serie de prostatectomías radicales retropúbicas (PRR), para intentar determinar si el tiempo de espera entre la biopsia y la PRR puede influir en la evolución bioquímica precoz (en el primer año post-prostatectomía). Según íbamos realizando el trabajo encontramos que otros factores como el Gleason de la biopsia, el PSA antes de la cirugía, el Gleason del espécimen quirúrgico, y el estadio pT final de la pieza suponen un impacto importante en esta mala evolución precoz.

\section{MATERIAL Y MÉTODOS}

Realizamos una revisión retrospectiva de las historias clínicas de 310 PRR realizadas en nuestro servicio entre Enero de 1995 y Diciembre de 2004 por cáncer de próstata localizado (T1c, T2 o T3a), que cumplían los siguientes criterios de inclusión: al menos un año de seguimiento, no manipulación hormonal ni radioterapia previa. En nuestro Servicio esta cirugía es realizada por todos sus miembros sin excepción lo que nos permite mostrar una serie heterogénea debido a la diferencia en experiencia quirúrgica. El seguimiento protocolizado en nuestro servicio tras PRR es: 3 meses postcirugía, 6 meses, un año, y luego anualmente, con PSA y examen físico que incluye tacto rectal. Ningún paciente recibió tratamiento hormonal ni radioterapia tras cirugía en el primer año. Este estudio pretende evaluar sólo el primer año de seguimiento. El estadio pT se estableció según la clasificación TNM de 1997. Los pacientes fueron divididos en dos gru- pos según tiempo de espera entre biopsia y prostatectomía radical. Grupo A < 90 días, Grupo B > 90 días. Tomamos 90 días como referencia porque la mediana de la serie es de 89,6 días. La asignación a uno $\mathrm{u}$ otro grupo se realiza por la propia lista de espera de nuestro sistema público, sin aleatorización. Estudiamos recurrencia bioquímica precoz (RBP) (2 o más determinaciones de PSA >=0,2 ng/ml en el primer año). The AUA Prostate Guideline Update Panel define la recurrencia bioquímica tras prostatectomía radical cuando un PSA sérico es de 0,2 ng/ml o superior con una segunda confirmación de un valor de PSA > 0,2 ng/ml. El primer PSA postoperatorio se debe determinar entre 6 semanas y 3 meses tras la operación. Se considera fecha de recurrencia como la primera obtenida después de la confirmación ${ }^{1}$.

Son también evaluados el impacto del tiempo de espera (TE), PSA previo, Gleason de la biopsia, Gleason del espécimen, y estadio T final (pT). Para determinar la homogeneidad entre los grupos en edad, PSA previo y Gleason de biopsia utilizamos la $\mathrm{T}$ Student o la W Wilcoxon. Para comparar RBP y pT en cada grupo, así como RBP según pT en la serie global usamos la Chi cuadrado de Pearson. Para estudiar la RBP según el Gleason del espécimen usamos la U Mann Whitney. Finalmente, para estudiar el impacto de todas las variables sobre la RBP usamos la Regresión logística multivariante.

\section{RESULTADOS}

Estudiamos 310 PRR y las dividimos en dos grupos. Grupo A (<90 días): n: 148. Edad: 62,2 años (R: 49-71), PSA medio pre-cirugía: 9,5 ng/ml (R: 3,219), Gleason de biopsia: 5.6 (R: 3-8). Grupo B (>90 días): n: 162. Edad media: 63.1 (R: 50-70), PSA medio pre-cirugía: 10,1 (R: 2,4-20), Gleason de biopsia: 5,6 (R: 2-8). Existía homogeneidad entre los dos grupos en lo que se refiere a edad $(\mathrm{p}<0,129)$, PSA ( $\mathrm{p}<0,479$ ), Gleason de biopsia ( $<<0,913$ ).

Recurrencia bioquímica precoz y pT según el retraso de la cirugía. En el Grupo A se produjeron 36 recurrencias bioquímicas en el primer año $(24,3 \%$ del grupo). En el Grupo B se produjeron 34 (20,7\%). En lo referente al estadio pT consideramos solamente los T2 y T3 porque no tenemos T4 en nuestra serie. En Grupo A observamos 90 T2 y 58 T3. En Grupo B 88 T2 y 76 T3. Cuando comparamos la 
recurrencia bioquímica y el pT entre los dos grupos usando la Chi Cuadrado de Pearson, no observamos diferencias significativas, lo que significa que en nuestra serie el tiempo de espera (TE) no parece influir en la recurrencia bioquímica en el primer año y tampoco en el estadio pT (Tablas 1 y 2).

Tabla 1

\begin{tabular}{lcc}
\hline RBP/TE & Grupo A & Grupo B \\
\hline $\mathrm{N}^{\circ}$ RBP & $36(24,3 \%)$ & $34(20,7 \%)$ \\
\hline
\end{tabular}

Grupo A versus Grupo B. Valor de $\mathrm{p}=0,554$.

Tabla 2

\begin{tabular}{lcc}
\hline pT/TE & Grupo A & Grupo B \\
\hline pT & T2 $90(60,8 \%)$ & T2 $88(53,6 \%)$ \\
& T3 $58(39,2 \%)$ & T3 $76(46,4 \%)$
\end{tabular}

Grupo A versus Grupo B. Valor de $\mathrm{p}=0,221$.

Recurrencia bioquímica precoz en relación al estadio pT y al Gleason del espécimen. Hallamos diferencias estadísticamente significativas en recurrencia bioquímica precoz atendiendo al estadio pT. De todos los pacientes sometidos a PRR que sufrieron recurrencia bioquímica en el primer año de seguimiento, 19 eran T2 y 52 eran T3, y entre aquellos que no tuvieron esta mala evolución, 158 eran T2 y 81 eran T3 (Tabla 3). Para estudiar la relación entre el Gleason final de la pieza y la recurrencia bioquímica precoz usamos el test no paramétrico de la $U$ de Mann-whitney porque la muestra no seguía una distribución normal. Encontramos en este caso que la posibilidad de recurrencia bioquímica precoz se incrementa al aumentar el grado de Gleason del espécimen (Tabla 4).

Regresión logística de la serie global. Utilizamos la regresión logística para evaluar todas las variables con el objeto de mostrar cuales pueden ser variables independientes para progresión. El PSA parece ser una de esas variables independientes. Como se puede observar el PSA elevado conlleva

Tabla 3

\begin{tabular}{lcc}
\hline $\mathbf{R B P} / \mathbf{p T}$ & $\mathbf{R B P}$ & No $\mathbf{R B P}$ \\
\hline $\mathbf{p T}$ & T2 $19(10,7 \%)$ & T2 $158(89,3 \%)$ \\
& T3 $52(39 \%)$ & T3 $81(61 \%)$ \\
\hline
\end{tabular}

RBP versus No RBP. Valor de $\mathrm{p}=0,000$.
Tabla 4

\begin{tabular}{cc}
\hline Gleason de pieza & n \\
\hline 3 & 2 \\
4 & 6 \\
5 & 30 \\
6 & 83 \\
7 & 164 \\
8 & 23 \\
9 & 2 \\
\hline
\end{tabular}

RBP es más frecuente según aumenta el Gleason.

Valor de $\mathrm{p}=0,028$

una probabilidad mayor de recurrencia bioquímica en el primer año, independientemente de las otras variables. De igual forma el estadio $\mathrm{T}$ final parece ser variable independiente. Los T3 tienen más riesgo de recurrencia bioquímica precoz que los T2, independientemente del resto de variables. El Gleason de la biopsia, el Gleason del espécimen, y el tiempo de espera no parecen influir de forma independiente en la recurrencia bioquímica precoz en la regresión logística (Tabla 5).

\section{DISCUSIÓN}

Tras realización de la biopsia de próstata suele esperarse entre 6 y 8 semanas para practicar una prostatectomía radical, para permitir que disminuya la inflamación local, lo que nos lleva a un abordaje quirúrgico teóricamente preciso, con menor morbilidad $^{2}$. Sin embargo no es extraño que la cirugía radical se retrase varios meses por diferentes razones. Dado que el carcinoma de próstata órgano confinado es susceptible de ser tratado con excelentes resultados de muy diversas formas (cirugía, radioterapia externa, braquiterapia, etc.), los pacientes deben ser educados sobre su enfermedad, conociendo los beneficios y riesgos de cada abordaje y decidiendo el mismo, lo que les lleva a veces a solicitar varias opiniones. Otro caso lo suponen países como el nuestro cuyo sistema público genera listas de espera largas, también en tumores genitourinarios. Cuando este retraso en el tratamiento es debido a la última causa se suele generar una ansiedad en el paciente que conoce su enfermedad y que estima que puede seguir avanzando en el periodo de espera.

Diversos estudios ha abordado el tema de si el tiempo de espera entre biopsia y prostatectomía radical influye en la recurrencia de la enfermedad, así como que factores afectan a la misma. 


\begin{tabular}{lccc}
\hline & Odds Ratio & CI 95\% & Valor de p \\
\hline PSA previo & 1,09 & $1,038-1,151$ & $=0,001^{*}$ \\
pT & 0,162 & $0,081-0,326$ \\
Gleason de biopsia & 1,051 & $0,753-1,467$ & $<0,001^{* *}$ \\
Gleason de pieza & 0,839 & $0,574-1,225$ \\
TE & 1,521 & $0,810-2,856$ & $<0,363$ \\
\hline
\end{tabular}

*El PSA elevado lleva a una mayor probabilidad de RBP independientemente de otras variables. **T3 tiene más riesgo de RBP que los T2 independientemente del resto de variables. ***No impactan en RBP

$\mathrm{Khan}^{2}$ en estudia $926 \mathrm{PRR}$, de las cuales 162 fueron realizadas dentro de los 60 días post biopsia y 764 posterior a 60 días. Compara edad, PSA previo, Gleason de la biopsia, estadios clínico y patológico y recurrencia bioquímica. Los operados entre 121 y 150 días, así como aquellos operados después de los 150 días eran con más frecuencia T1c que aquellos operados antes de 60 días. Asimismo más hombres operados después de los 150 días tenían Gleason 2-6, en relación también a aquellos operados antes de 60 días. Aquellos pacientes sometidos a PRR entes de los 60 días tenían la misma probabilidad de recurrencia que aquellos sometidos a PRR entre 61 y 90, 91 y 120, 121 y 150 días tras diagnóstico. Aquellos sometidos a PRR tras 151 días tenían tasas de supervivencia libre de enfermedad superior a los 5 y 10 años. Este estudio concluye que el retraso desde el diagnóstico hasta la PRR no parece influir en el control oncológico. Parece claro que aquellos pacientes con Gleason $<7$ y enfermedad T1c pueden esperar hasta varios meses.

Datos similares aporta el monumental estudio retrospectivo del Memorial Sloan Kettering Center ${ }^{3}$ revisando 3.149 PRR consecutivas. Para pacientes operados en los primeros 6 meses la odds ratios fue de $1,04,1,07,1,08$, y 1,02 respectivamente para 3 , $5,8$ y 10 años libre de enfermedad ( $>0,2)$. Por tanto el tiempo entre biopsia y recurrencia no parece tener un efecto en la recurrencia.

Graefen ${ }^{4}$ estudia 795 pacientes, definiendo recurrencia como PSA > 0,1 ng/ml. Tampoco encuentra diferencias significativas en recurrencia según tiempo hasta intervención, y si en relación al PSA previo, estadio clínico y Gleason de la biopsia. Datos similares arroja el estudio de Shibata ${ }^{5}$ que encuentra relación estadísticamente significativa entre la aparición de recurrencia y el PSA previo y el Gleason de la prostatectomía radical, sin hallar relación de di- cha recurrencia con el tiempo de espera. Ambos estudios coinciden con nuestro análisis. Verdaderamente parece que los parámetros implicados en la recurrencia son los datos analíticos, y clínico-patológicos y no el tiempo de espera.

Mención especial merece el estudio de Lee ${ }^{6}$ acerca de la influencia del tiempo de espera sobre la evolución postoperatoria inmediata. Se analizan márgenes positivos, necesidad de transfusiones, complicaciones postoperatorias, continencia y preservación de bandeletas, y recurrencia bioquímica. En el análisis de regresión logística y lineal y tras ajustar posibles factores de confusión (Gleason de la pieza, PSA y edad), no se observaron diferencias significativas entre el tiempo de espera y ninguna de las variables a estudio. Se concluye por tanto que el intervalo entre biopsia y prostatectomía radical no parece afectar la evolución postoperatoria inmediata.

Sin embargo podríamos preguntarnos si todos los pacientes pueden o no esperar. Es decir, según las características del tumor (PSA previo, Gleason de biopsia o estadio clínico) ¿habría algunos (alto riesgo) en los que si influiría la espera?. La serie del Memorial en otro trabajo $^{7}$ nos muestra que ni siquiera en aquellos casos considerados de alto riesgo para recidiva (PSA $>=20$, Gleason de biopsia $>=$ 8 , cT $>=2$ c) parece influir el tiempo de espera. Sin embargo como ya expusimos antes y era uno de los objetivos de nuestro estudio si se muestra que las características clínicas y patológicas son los mejores predoctores de recidiva. A pesar de la evidente validez de estos trabajos, existen otros autores que si encuentran diferencias. Un estudio de la Universidad de Toronto $^{8}$ encuentra que a 10 años la supervivencia es mayor en el grupo de cirugía más precoz, así como los índices de recurrencia bioquímica lo son en el grupo de cirugía tardía. Parecería que puede existir una relación entre el retraso en el tratamiento 
y los índices de curación. Asimismo parece que el tiempo óptimo de espera debería ser menos de 3 meses post biopsia sobre todo en aquellos pacientes de alto riesgo. Otra vez los factores pronósticos de recurrencia serían extensión extracapsular, margen quirúrgico y afectación de vesículas. Ajustando por estas 3 variables el retraso en la cirugía estaría relacionado con recurrencia ${ }^{9,10}$.

El PSA inmediatamente previo a PRR y el Gleason en la pieza de PRR son los principales predictores de recurrencia bioquímica, mientras que el tiempo entre biopsia y PRR, la variación de PSA y Gleason desde la biopsia hasta la PRR, el PSA pre biopsia, y el Gleason de la biopsia no son predictores de recidiva.

\section{REFERENCIAS}

1. Thompson I, Thrasher JB, Aus G, Burnett AL, Canby-Hagino ED, Cookson MS, et al. Guideline for the management of clinically localized prostate cancer: 2007 update. J Urol. 2007;177 (6):2106-2131.

2. Khan MA, Mangold LA, Epstein JI, Boitnott JK, Walsh PC, Partin AW. Impact of surgical delay on long term cancer control for clinically localized prostate cancer. J Urol. 2004;172(5):18351839.

3. Vickers AJ, Bianco FJ Jr, Boorjian S, Scardino PT, Eastham JA. Does a delay between diagnosis and radical prostatectomy increase the risk of disease recurrence?. Cancer. 2006;106(3):576580 .
4. Graefen M, Walz J, Chun KH, Schlomm T, Haese A, Huland H. Reasonable delay of surgical treatment in men with localized prostate cancer impact on prognosis?. Eur Urol. 2005;47(6): 756-760.

5. Shibata A, Mohanasundaram UM, Terris MK. Interval from prostate biopsy to radical prostatectomy: effect on PSA, Gleason sum, and risk of recurrence. Urology. 2005;66(4): 808-813.

6. Lee DK, Allareddy V, O'donnell MA, Williams RD, Konety BR. Does the interval between prostae biopsy and radical prostatectomy affect the immediante postoperative outcome?. Br J Urol Int. 2006;97(6):48-50.

7. Boorjian SA, Bianco FJ Jr, Scardino PT, Eastham JA. Does the time from biopsy to surgery affect biochemical recurrence after radical prostatectomy?. Br J Urol Int. 2005;96(6): 773-776.

8. Nam RK, Jewett MA, Krahn MD, Robinette MA, Tsihlias J, Toi A, et al. Delay in surgical therapy for clinicallly localized prostate cancer and biochemical recurrence after radical prostatectomy. Can J Urol. 2003;10(3):1891-1898.

9. Moul JW, Sun L, Amling, C, et al. How long can radical prostatectomy (RP) safely be delayed? CPDR's experience from 3324 cases. J Urol. 2004;171(suppl):312.

10. Mohanasundaram, U.; Shibata, A.Time interval from prostate biopsy to prostatectomy: relationship to change in PSA and Gleason grade from biopsy to surgery and correlation with biochemical recurrence. J Urol, 2004;171(suppl):278.

Correspondencia autor: Dr. A. Blanco Díez

Servicio de Urología. Hospital Universitario Insular de Gran Canaria Pza. del Doctor Pasteur, s/n - 35016 Las Palmas de Gran Canaria. Tel.: 928444000

E-mail autor: abdiez2003@yahoo.es

Información artículo: Original - Cáncer de Próstata

Trabajo recibido: octubre 2007

Trabajo aceptado: noviembre 2007 